\title{
Intestinal Stem Cells Isolation and Culture Characteristics in vitro by Morphometry and Flow Cytometry
}

\author{
K. Kaviyarasi ${ }^{1 *}$, A. Mangala Gowri ${ }^{2}$, T.M.A. Senthilkumar ${ }^{3}$, \\ S. Hemalatha ${ }^{4}$ and K. Vijayarani ${ }^{5}$ \\ ${ }^{1}$ Department of Animal Biotechnology, ${ }^{3}$ Department of Animal Biotechnology, \\ ${ }^{4}$ Department of Veterinary Pathology, Madras Veterinary College, Ch- 7. TN, India \\ ${ }^{2}$ Department of Animal Biotechnology and Centre for Stem Cell Research and Regenerative \\ Medicine, Tamil Nadu Veterinary and Animal Sciences University, Ch- 7. TN, India \\ ${ }^{5}$ Department of Animal Biotech, MVC, TANUVAS, Ch-7 \\ *Corresponding author
}

\begin{abstract}
A B S T R A C T
Adult stem cells are crucial for maintaining proper function and repair of tissues. The epithelium of the adult mammalian intestine is a constant dialog with its underlying mesenchyme to direct progenitor proliferation, lineage commitment, terminal differentiation and ultimately cell death. The epithelium is shaped into spatially distinct compartments that are dedicated to each of these events. The unique intestinal orchestration makes the crypt as one of the most accessible models for the study of adult stem cell biology (Barker et al., 2007). Because intestine is one of the most rapidly regenerated tissues in the body, the intestinal crypt has provided an informative system for studying stem cell biology. The full potential of these models was not realized, however, duo to the limited availability of optimized protocols and unique stem cell markers to identify the location and numbers of ICSs as well functional assays to validate the models in vivo. Though Intestinal stem cell represented by crypt population has been established in mice and human but the understanding of the underlying mechanism was not reported in other animal species in detail. The present research focussed on the isolation of intestinal crypts and their in vitro culture characteristics in chicken, a mammalian animal model widely used for research and food application has been carried out using cytochemical and fluorescent activated cell sorting for stem cell specific marker Sox 9 .
\end{abstract}

\section{Keywords}

Intestinal stem cells,

Crypts, Chicken, Isolation and culture, Flow

cytometry

Article Info

Accepted:

26 April 2018

Available Online:

10 May 2018

\section{Introduction}

In vitro cell models are one of the validated options for the replacement of live animal methods. They allow the screening of specific mechanisms of epithelial permeability, potentially in a high throughput manner. They present a more economic and ethical alternative to animal testing. The lack of a proper in vitro model system has hampered both the fundamental analysis of absorption biology of food bioactives or drug biomolecules in situ and applied research of intestinal tract biology. Accumulation of 
current knowledge on crypt stem cell behaviour has led to the hope on promising intestinal cell culture approaches to design models to explore bioactives cellular interaction. The main advantage of intestinal cell models is their simplicity, inter-laboratory repeatability and large-scale testing capacity. Functional intestinal cell models hold several advantages over generic cell lines, as they can simulate more closely the in vivo environment, without however sacrificing their simplicity and reliability (i.e., 2005). The epithelium of the adult mammalian intestine is in a constant dialog with its underlying mesenchyme to direct progenitor proliferation, lineage commitment, terminal differentiation, and ultimately, cell death. The unique epithelial anatomy makes the intestinal crypt one of the most accessible models for the study of adult stem cell biology (Baker et al., 2014).

\section{Materials and Methods}

Intestine samples from chicken were collected from the slaughter house in a sterile container in PBS along with antibiotic solution. Intestine separation from loose mesenchymal tissue and visible blood vessels for transfer and dissection were followed. From the collected sample, the proximal end of about $15 \mathrm{~cm}$ was washed several times with PBS and cut longitudinally, then the inner side of the intestine was washed several times with ice cold PBS. The crypt cells were isolated by a modified procedure from the intestine based on the isolation method by O'Rouke, et al., (2016). A modification of the isolation protocol with an extended incubation has been carried out. Epithelial tissue separation and standardization of protocols for isolation of crypts cell fractions was done. Representative isolated crypt cells were confirmed morphologically by staining. The isolated crypts were counted prior to culturing of the cells. The number of crypts plated in 96 well micro titre plate were distributed by limiting dilution and observed for morphological changes during different culture days. The cells were characterized for the expression of surface biomarkers - Sox9 by fluorescent activated cell sorter (S3 Sorter, Biorad) as stated by Furuyama et al., (2011)

\section{Results and Discussions}

Crypts were isolated (Fig. 1) separately from three regions of small intestine and the crypts yield from three sections were analysed. It was found that the duodenum has higher yield of crypts among three sections. The results are shown in Table 1. There was a significant gated population of SOX 9 positive individual crypt population has been identified in flow cytometry analysis (Fig. 2A and 2B)

The crypt to villus hierarchical migratory pattern of cell proliferation and differentiation is well established and it is therefore assumed that the stem cells are located at the origin of this system, the intestinal crypt. The stem cell compartment is proposed to be at the base of the colonic crypts and approximately at cell position 4-5 in small intestine. It is thought that stem cells reside within a stem cell compartment or niche. Also it has been reported that the crypts having more than one functional stem cell were reported to be derived from single stem cell (Ponder et al., 1985). Those stem cells were thought to reside within a niche- stem cell compartment (Powell, 1999) and were maintained by production versus cell deletion. The production was reported to be mainly based on asymmetrical division i.e., from one stem cell, a stem cell and a daughter cell (Lewis and Radtke, 2011). The strongest evidence for the stochastic nature of the division process has been shown to be leading to monoclonal conversation, and concludes that the cells in a crypt become descendant of a single stem cell (Zhang et al., 2016). 
Fig.1 Morphometry of isolated crypts cultured in matrigel culture (Scale bar 100 $\mu$ m)

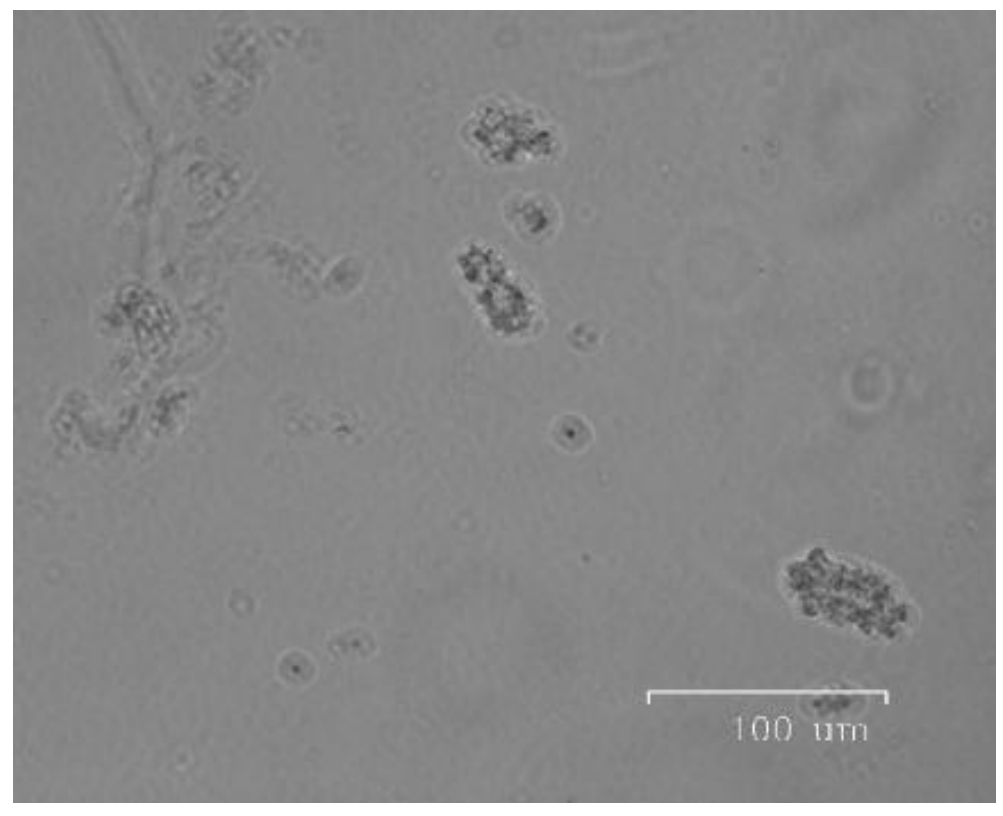

Fig.2 The flow cytometric analysis for SOX9 labelled cells

A

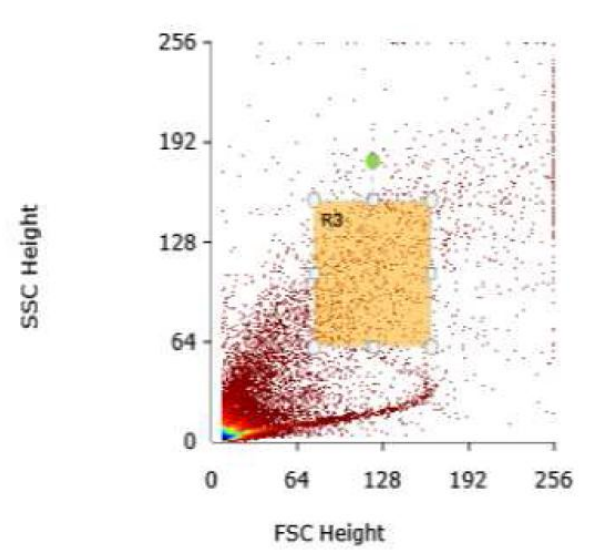

B

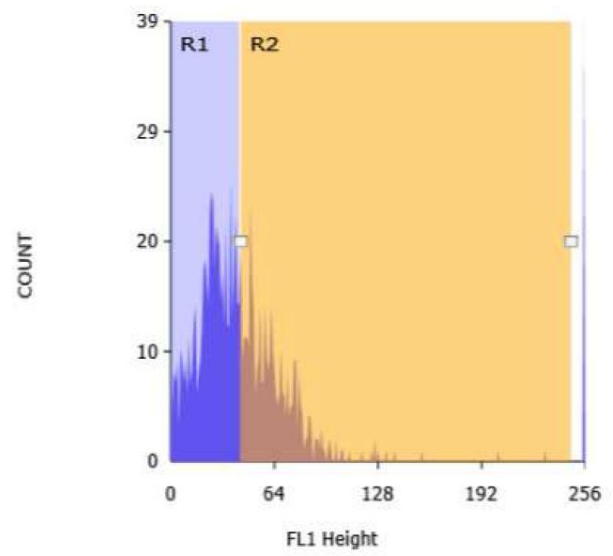

A Statistics
\begin{tabular}{|lcccccc|}
\hline Label & Count & Mean & $\%$ of Total & $\%$ of Plot & CV & Std Der| \\
\hline Total & 1000 & 49.34 & $2.92 \%$ & $100.00 \%$ & 97.23 & 47.97 \\
R1 & 551 & 24.52 & $1.61 \%$ & $55.10 \%$ & 43.85 & 10.75 \\
R2 & 410 & 63.13 & $1.20 \%$ & $41.00 \%$ & 32.34 & 20.42 \\
\hline
\end{tabular}

Table.1 Comparison on average crypt population in small intestine $(\mathrm{N}=18)$

\begin{tabular}{|l|c|}
\hline Chicken Small Intestine & No. of Crypts Observed per square millimeter (mean \pm \\
\hline Duodenum & SE) \\
\hline Jejunum & $\mathbf{5 9 . 8 3} \pm \mathbf{1 . 2}$ \\
\hline Ileum & $\mathbf{4 7 . 1 6} \pm \mathbf{0 . 9}$ \\
\hline
\end{tabular}


The present research finding on the higher yields of crypt population from duodenum than other locations of small intestine supports the above hypothesis and stated that the incubation time and process of crypt isolation plays a major role and varies with species to species.

Murine small intestine constitutes about an average of 250 cells in a test tube like structure, in longitudinal cross section they are approximately 22 cells in height with 16 cells in wide (Potten et al., 1990). In vitro experimental studies may alter the behaviour of stem cells, they are different estimates widely available for stem cell number (Potten and Loeffler, 1990). Isolation of crypts has been carried out by using the procedure standardized in mouse (O'Rouke et al., 2016). Modification has been made in order to make the isolation process suitable for chicken. Incubation period of intestinal sections has been increased to 20 minutes in order to harvest the intact crypts suitable for further applications whereas ten minutes incubation was sufficient in mouse which could be attributed to earlier reports on species variation.

A common feature of both the Wnt and Notch pathways is that they appear to be modulated by Sox (Sry-Box) genes (Formeister et al., 2009) indicating a critical role for SOX transcription factors in normal intestinal epithelium homeostasis. SOX transcription factors have the capacity to modulate stem/progenitor cell proliferation and differentiation in a dose-dependent manner. SOX9 is expressed in the small intestine epithelial stem cell zone. Sox genes are a family of at least 20 closely related transcription factors, which are defined by the presence of a high-mobility group domain. All Sox factors bind a relatively loose consensus sequence, resulting in dramatic DNA bending that has been hypothesized to be critical for bringing distal control elements to proximal transcriptional start sites. SOX factor competition for a single cis-control element combined with the physical interaction of SOX factors with other transcription factors influences the specificity of target gene transcription. Because distinct levels of SOX factors appear to be critical for proper proliferation and differentiation in stem cell biology, it could be hypothesized from the present research findings that the differential SOX9 expression may be playing a similar role in stem/progenitor cell populations in the intestinal epithelium. Furuyama et al., (2011) showed that Sox9 was expressed throughout the biliary and pancreatic ductal epithelia, which were connected to the intestinal stem-cell zone. Cre-based lineage tracing showed that adult intestinal cells, hepatocytes and pancreatic acinar cells were supplied physiologically from Sox9-expressing progenitors.

In the present research intestinal crypt population are isolated and their identity for stem cells were shown using expression of sox 9 a candidate embryonic stem cell marker.

Hence the crypt cells could be in vitro cultured and applied in drug testing / discovery approaches with more application of molecular tools.

\section{Acknowledgement}

Authors acknowledge the Centre for stem cell research and regenerative medicine, Madras Veterinary College, TANUVAS for the facilities provided to carry out this research.

\section{References}

Barker, N., J.H. van Es, J. Kuipers, P. Kujala, M. van den Born, M. Cozijinsen, A. Haegebarth, J. Korving, H. Begthal, P.J. 
Peters and H. Clevers, 2007. Identification of stem cells in small intestine and colon by marker gene Lgr5. Nature, 449: 1003-1007.

Formeister. E.J, Sionas. A.L, and Scott T. Magness, 2009. Distinct SOX9 levels differentially mark stem/progenitor populations and enteroendocrine cells of the small intestine epithelium. Am $J$ Physiol Gastrointest Liver Physiol. 296: 5

Furuyama. K, Kawaguchi. Y, Akiyama. H and Horiguchi. M. 2011. Continuous cell supply from a Sox9-expressing progenitor zone in adult liver, exocrine pancreas homeostasis of intestinal stem cells. Gastroenterology, 140: 12301240.

Lewis and Radtke. 2011. DII1- and dII4mediated notch signalling are required for homeostasis of intestinal stem cells. Gastroenterology, 140: 1230-1240

Moore, K.A. and I.R. Lemischka, 2006. Stem cells and their niches. Science, 311:1880-1885.
O’Rouke, et al., (2016). O'Rouke, K.P., S. Ackerman, L.E. Dow and S.W. Lowe. 2016. Isolation, culture and maintenance of Mouse intestinal stem cells. Bioprotocol. 6: e1733.

Ponder, B.A., G. Schmidt and M.M. Wilkinson. 1985. Derivation of mouse intestinal crypts from single progenitor cells. Nature, 313: 689-691.

Potten, C. S., G. Owen and S.A. Roberts. 1990. The temporal and spatial changes in cell proliferation within the irradiated crypts of the murine small intestine. Int. J. Radiat. Biol., 57, 185-199.

Potten, C.S., and M. Loeffler. 1990. Stem cells: attributes, cycles, spirals, pitfalls and uncertainties. Lessons for and from the crypt. Development, 110: 10011020.

Zhang Y, E. Viennois, M. Zhang, B. Xiao, M.K. Han, L. Walter, P. Garg, D. Merlin, 2016. PepT1 Expression Helps Maintain Intestinal Homeostasis by Mediating the Differential Expression of miRNAs along the Crypt-Villus Axis. Sci Rep, 6: 271-19.

\section{How to cite this article:}

Kaviyarasi, K., A. Mangala Gowri, T.M.A. Senthilkumar, S. Hemalatha and Vijayarani, K. 2018. Intestinal Stem Cells Isolation and Culture Characteristics in vitro by Morphometry and Flow Cytometry. Int.J.Curr.Microbiol.App.Sci. 7(05): 3731-3735. doi: https://doi.org/10.20546/ijcmas.2018.705.432 